\title{
Modification of a Popular Syngeneic Murine Mammary Tumor Model for Immunotherapy Studies
}

\author{
Armando Rivera, ${ }^{1,2}$ Xinping Fu, ${ }^{1,2}$ Lihua Tao,, ${ }^{1,2}$ and Xiaoliu Zhang, \\ ${ }^{1}$ Department of Biology and Biochemistry, University of Houston, 4800 Calhoun Road, Houston, TX 77204, USA \\ ${ }^{2}$ Center for Nuclear Receptors and Cell Signaling, University of Houston, Houston, TX 77204, USA \\ Correspondence should be addressed to Xiaoliu Zhang, shaunzhang@uh.edu
}

Received 21 May 2011; Accepted 22 June 2011

Academic Editors: A. Vicente and A. Vyakarnam

Copyright (C) 2011 Armando Rivera et al. This is an open access article distributed under the Creative Commons Attribution License, which permits unrestricted use, distribution, and reproduction in any medium, provided the original work is properly cited.

\begin{abstract}
To facilitate the evaluation of immunotherapeutic intervention against malignant diseases, it is desirable to have a syngeneic tumor model that closely resembles the growth pattern of human tumors. Murine 4T1 breast cancer model is known for its metastatic properties that mimic its human counterpart. However, a drawback of this model is the lack of an identified tumor antigen to function as a therapeutic target for immunologic intervention. We used the piggyBac transposon system to stably transduce a tumor antigen, the human epidermal growth factor receptor 2 gene (HER2), into this tumor cell. In vitro characterization shows that the newly established cells have a similar growth pattern as the parental line. In vivo evaluation shows that host immune response was generated against the HER2 tumor antigen, despite the high homology between HER2 and its murine counterpart (neu gene). When implanted into immune-deficient mice, the HER2-expressing 4T1 cells readily formed sizable tumors, indicating that these cells are useful for evaluating the therapeutic effect of adoptively transferred cytotoxic $\mathrm{T}$ cells that are specifically raised or modified to target the HER2 tumor antigen.
\end{abstract}

\section{Introduction}

The benefit of preclinical tumor models for evaluating cancer therapeutics depends largely on whether the tumors growing in animals closely mimic the characteristics of the human counterpart. The most frequently used preclinical models are those from tumor cell implantation, as they are easily controlled as compared with chemically induced tumors or those developed from genetic alterations. Depending on the source of the tumor cells, tumors could either be xenograft or syngeneic. While xenografted tumors must be grown in immune deficient animals, syngeneic tumors are grown in animals whose immune systems are intact. This is an especially desirable characteristic since the efficacy of many cancer therapeutics can only be truly evaluated in the presence of an intact host immune function.

Many implanted tumors do not metastasize naturally [1]. The 4T1 murine breast cancer cell line, however, is well known for its ability to metastasize to different organs, such as lung, liver, brain, and bones [2]. More importantly, the metastatic pattern of $4 \mathrm{~T} 1$ tumor model closely mimics that of human breast cancer. Metastases of 4T1 tumor cells are efficient and usually occur within 2-4 weeks after tumor implantation; consequently, the $4 \mathrm{~T} 1$ murine breast cancer model is a valid, useful tumor model and has been widely used in studies for evaluating antitumor effects of many cancer therapeutics [3], especially those designed for immunologic interventions $[4,5]$.

Unlike other commonly used syngeneic tumor models such as B16 murine melanoma, which contains wellcharacterized tumor antigens such as gp100 [6], tumorassociated antigens in 4T1 tumor cells have not been clearly defined. This has severely limited the usefulness of this tumor model despite its natural metastatic pattern that closely mimics the human disease. Here we report our effort in modifying $4 \mathrm{~T} 1$ cells by stably introducing the human epidermal growth factor receptor-2 (HER2, ErbB-2) gene into the cells. HER2 is a tumor-associated antigen and is over-expressed in many malignant cells, especially in breast and ovarian cancer cells. Its overexpression is also frequently associated with 
aggressive tumor growth and invasiveness [7]. Unlike many identified tumor-associated antigens, HER2 is expressed on the surface of tumor cells. Thus, it represents a good target for anticancer immunotherapy [8]. Using a piggyBac transposon system, we have established several 4T1 cell lines that highly express HER2. We then fully characterized these cell lines in both in vitro and in vivo studies. Our data suggests that the introduced tumor antigen indeed can generate immune responses and can function as a target for evaluating immunologic interventions.

\section{Material and Methods}

2.1. Cell Lines and Cell Culture. 4T1 cells, a 6-thioguanineresistant cell line derived from a BALB/c spontaneous mammary carcinoma [2], were kindly provided by Dr. Fred Miller (Michigan Cancer Foundation, Detroit, MI, USA). Mouse breast tumor cell line D2F2/E2, which was derived from a spontaneous mammary tumor and over-expresses HER2 [9], was a gift from Dr. Wei-Zhen Wei (Wayne State University). All cell lines were maintained in DMEM (Invitrogen, Carlsbad, CA) and supplemented with $10 \%$ fetal bovine serum (FBS, purchased from Gemini Bioproducts, West Sacramento, CA), 100 units/mL penicillin, and $100 \mu \mathrm{g} / \mathrm{mL}$ streptomycin (Invitrogen). Cells were incubated at $37^{\circ} \mathrm{C}$ in a humidified atmosphere saturated with $5 \% \mathrm{CO}_{2}$.

2.2. Chemicals and Antibodies. Monoclonal antibody against erbB2/HER-2 (324405) was purchased from BioLegend. A rabbit polyclonal antibody (ab2428) and a monoclonal antiactin antibody (Ab3280) were purchased from Abcam (Cambridge, MA). pIR-PURO plasmid was purchased from Invitrogen. Concanavalin A (C7275), Puromycin (P9620), and amphotericin B (A2942) were purchased from SigmaAldrich. (St. Louis, MO). The tetrazolium compound MTS, [3-(4,5-dimethylthiazol-2-yl)-5-(3 carboxymethoxyphenyl)2-(4-sulfophenyl)-2H-tetrazolium, inner salt], the electron coupling reagent phenazine ethosulfate (PES) (CellTiter $96 \mathrm{AQ}_{\text {ueous }}$ One Solution Cat \# G3582), and Bright-Glo Luciferase Assay System were purchased from Promega (Madison, WI). The Lipofectamine 2000 and Opti-MEM were products of Invitrogen. All the reagents were used as per manufacturer's instructions.

\subsection{Generation of HER2-Containing Vectors for Establishing} Stable Cell Lines. The HER2/neu WT plasmid was kindly provided by Dr. Mien-Chie Hung (M.D. Anderson Cancer Center). HER2/neu gene was excised and cloned into the mammalian expression delivery vector pIR-PURO. A fusion gene of GFP, and luciferase created in the lab was also cloned into another pIR-PURO plasmid (Figure 1). The details of plasmid pCMV-piggyBac have been described elsewhere [10]. It was transfected together with pIR-EGFP-Luc or pIREGFP-Luc plus pIR-PURO-HER2/neu (Figure 1) into 75\% confluent $4 \mathrm{~T} 1$ cells in an equal ratio, using Lipofectamine 2000 and Opti-MEM. Puromycin was added to transfected cells $48 \mathrm{~h}$ later at a concentration $1 \mu \mathrm{g} / \mathrm{mL}$, which was

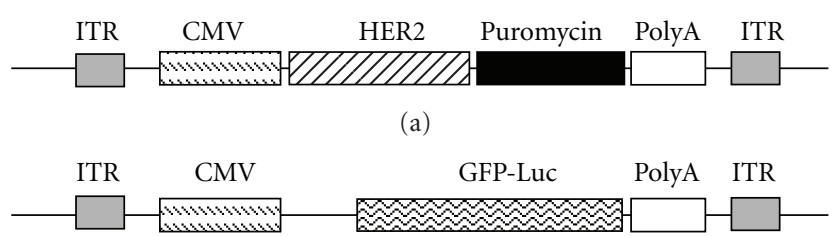

(b)

Figure 1: Schematic diagram of piggyBac constructs used to establish stable cell lines. (a) pIR-HER2-puromycin. This construct contains HER2 gene in addition to puromycin gene. It was used in cotransfections for making stable cell lines expressing HER2. (b) pIR-GFP-Luc. This construct contains the fusion gene of GFP and luciferase. It was used in all the co-transfections in which the GFPLuc fusion gene serves as a convenient marker for identifying the stably transfected cells. The locations of the promoter, the polyA signal, and the inverted terminal repeats (ITR) are indicated.

gradually increased up to $3 \mu \mathrm{g} / \mathrm{mL}$ over a course of two weeks.

The puromycin resistant cells expressing GFP were sorted by FACS analysis. The puromycin-resistant, GFP-, and HER2-expressing cells were confirmed by FACS analysis (GFP and HER2 double positive cells), using a monoclonal antibody specific for HER2. For the western blot analysis, cells seeded in 6-well plates were harvested and cell lysates were loaded to a 10\% SDS-PAGE. After electrophoresis, proteins were transferred to membrane for blotting. A rabbit polyclonal antibody (Abcam) to HER2 was used in a dilution of $1: 500$ and incubated at $4^{\circ} \mathrm{C}$ overnight. The corresponding band was revealed using the ECL Plus Western blot detection system (Amersham, Piscataway, NJ). After autoradiography acquisition, the membranes were stripped and reprobed for $2 \mathrm{~h}$ at room temperature with monoclonal anti-actin antibody (Abcam) to normalize protein loading.

2.4. Flow Cytometric Analysis and Cell Sorting. Monoclonal to HER2/neu-phycoerythrin, in a concentration of $20 \mu \mathrm{L}$ per million cells, was used for live sorting. Cells were stained in the dark with a PBS solution containing $2 \%$ fetal bovine serum (FBS), 100 units/mL penicillin-streptomycin, and $1 \mu \mathrm{g} / \mathrm{mL}$ amphotericin $\mathrm{B}$ for $30 \mathrm{~min}$ at $4^{\circ} \mathrm{C}$. After staining, cells were washed twice with the same buffer. FACS analysis was performed on a Becton Dickinson FACSAria (BD Biosciences). Gates were established with unstained cells operating at low pressure (20 psi) using a $100 \mu \mathrm{m}$ nozzle. Cell clusters and doublets were electronically gated out. Cells were routinely double-sorted using GFP and PE, and postsort analysis typically indicated purities of $>90 \%$ with minimal cell death $(<10 \%)$.

2.5. Transduction of Murine Splenocytes with a Retroviral Vector Containing Chimeric T-Cell Receptor. The construction of the retroviral vector (SFG-HER2/neu-CD28-Zeta) containing chimeric T-cell receptor (TCR) that specifically recognizes HER2/neu has been described elsewhere [11]. To produce the retroviral vector stock SFG-HER2/neu-CD28Zeta, PlatE cells were cotransfected with the packaging 
plasmid pCL-ECO (purchased from Imgenex, San Diego, CA) by using lipofectamine 2000. Supernatants containing the retrovirus were collected 48 and $72 \mathrm{~h}$ later and were used to transduce freshly harvested splenocytes from BALB/C mice. Briefly, splenocytes were harvested from BALB/C mice and cultured with RPMI 1640 medium supplemented with $25 \mathrm{mM}$ HEPES, $200 \mathrm{nM}$ L-glutamine, 10\% FBS, 1\% MEM nonessential amino acids, $1 \mathrm{mM}$ sodium pyruvate, $50 \mu \mathrm{M} \beta$-mercaptoethanol, $100 \mu \mathrm{g} / \mathrm{mL}$ streptomycin, and $100 \mathrm{U} / \mathrm{mL}$ penicillin. Single-cell suspensions $\left(2 \times 10^{6} / \mathrm{mL}\right)$ were activated with Concanavalin A $(2 \mu \mathrm{g} / \mathrm{mL})$ for $24 \mathrm{~h}$. The stimulated cells were transduced with HER2/neu-CD28Zeta retrovirus supernatant in a 24-well nontissue culture plate precoated with RetroNectin (Takara Bio. Inc. Shiga, Japan). Retroviral transduced splenocytes were then cultured for 48 hours in fresh medium supplemented with mIL-2 $(10 \mathrm{ng} / \mathrm{mL})$ to allow the cells to recover. They were then used directly for T-cell-mediated cytotoxicity assay.

2.6. In Vitro Cell Proliferation Assay. Five single-cell colonies (C1-C5) that were puromycin-resistant and were confirmed to express GFP-Luc and HER2 were chosen for this in vitro assay. The parental 4T1 and the 4T1-derived cells expressing GFP-Luc only were included as controls. Cell proliferation was measured using a colorimetric assay. Briefly, cells were seeded at 1000 cells/well in 96-well plates. Growth was evaluated at $24,48,72,96$, and $120 \mathrm{~h}$ later by adding the MTS reagent to the wells. The quantity of formazan product was determined with a colorimeter. The experiments were performed in triplicate and repeated at least 3 times.

2.7. In Vitro Cell Invasion Assay. The extracellular matrix invasion assay was performed essentially as described [12]. Briefly, the 24-well BioCoat Matrigel invasion chambers with $8-\mu \mathrm{m}$ pore size coated with Matrigel $(0.4 \mu \mathrm{g} / \mathrm{mL})$ (BD Biosciences) were rehydrated according to the manufacturer's instructions. Cells were suspended in serum free mediaDMEM and $2.5 \times 10^{4}$ cells were seeded in each well in the insert. The lower chamber was filled with media containing either $1 \%$ or $10 \%$ FBS. The cells were incubated for $22 \mathrm{~h}$ at $37^{\circ} \mathrm{C}$ in a $5 \% \mathrm{CO}_{2}$ atmosphere. The nonmetastatic cells on the insert upper surface were removed with a cotton swab. Metastatic cells clinging at the bottom of the membrane were fixed in methanol and stained with Hoechst 33258 (300 nM). The number of invasive cells was determined by counting five adjacent microscope fields at 20x magnification. The assays were performed in triplicate and repeated at least 3 times.

2.8. Anchorage-Independent Growth Assay. Anchorage-independent cell growth was determined based on colony formation in soft agar. Briefly, cell suspensions (at a final concentration of 2000 cells/well) were mixed with $0.35 \%$ agar solution and seeded over $0.7 \%$ agar layer. Cells were incubated at $37^{\circ} \mathrm{C}$ for 20 days. In order to avoid desiccation and to allow continuous growth, $20 \mu \mathrm{L}$ of fresh medium was added every 3 days. At the end of the experiment, the plates were stained with $0.5 \mu \mathrm{g} / \mathrm{mL}$ Hoechst 33258 for $30 \mathrm{~min}$. Colonies $>0.1 \mathrm{~mm}$ in diameter were counted using five adjacent microscope

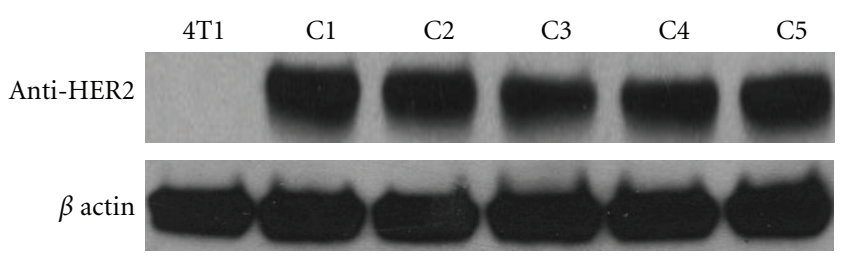

FIGURE 2: HER2 expression detected by Western blot. Proteins were extracted from cells and loaded to a 10\% SDS-PAGE. The western blot was done using a rabbit polyclonal antibody to HER2. Western blot for actin was used as a loading control.

fields at 20x magnification. The assays were performed in triplicate and repeated at least 3 times.

\subsection{Cytolytic Activity of Chimeric T-Effector Cells against} HER2-Expressing 4T1 Cells. Splenocytes that were transduced with the chimeric T-cell receptor were seeded with HER2-expressing or control cells at an $1: 1$ ratio in triplicate into 96-well plates. The cytotoxicity was quantitated by a nonradioactive method recently developed in our own lab [13]. Briefly, immediately before the assay for luciferase activity, culturing medium was removed. A mixture of $50 \mu \mathrm{L}$ of the assay luciferase reagent and $50 \mu \mathrm{L}$ of PBS was added to each well. Fifty $\mu \mathrm{L}$ of the lysate was then transferred to an opaque 96-well flat-bottom microtiter plate (Corning, Corning, NY) for luminescence measurements. Bioluminescence was detected with a SpectraMaxM5 MultiMode microplate reader using SoftMax Pro5 (Molecular Devices, Sunnyvale, CA). The assays were carried out in triplicate and repeated at least 3 times.

2.10. Animal Experiments. All animal experiments were carried out at the Animal Care Operations facility of the University of Houston, Houston, Texas, and approved by the University of Houston Institutional Animal Care and Use Committee (IACUC). Tumor cells at a dose of $1 \times 10^{5}$ cells were implanted into the mammary fat pad around the fourth right nipple of 6-8-week female immunocompetent BALB/C mice (Charles River, Wilmington, MA) or immunodeficient mice (Taconic, Hudson, NY). There were 5 mice per group. Once tumor became palpable, they were measured every three days until the end of the experiment. Tumor size was determined using a vernier caliper by measuring the largest and the smallest diameters of the tumor. Tumor volumes were calculated according to the formula $V=$ length $\times$ $(\text { width })^{2} \times 0.5$. Tumor challenge with the D2F2/E2 cells was performed with the same procedure.

2.11. Statistical Analysis. All the data were statistically analyzed using Student's $t$-test for pairwise comparison. $P<0.05$ was considered significant.

\section{Results and Discussion}

3.1. Establishment of Stable 4T1 Cell Lines Expressing HER2. To facilitate the establishment of stable integration of HER2 into 4T1 tumor cells, we initially cloned the gene 


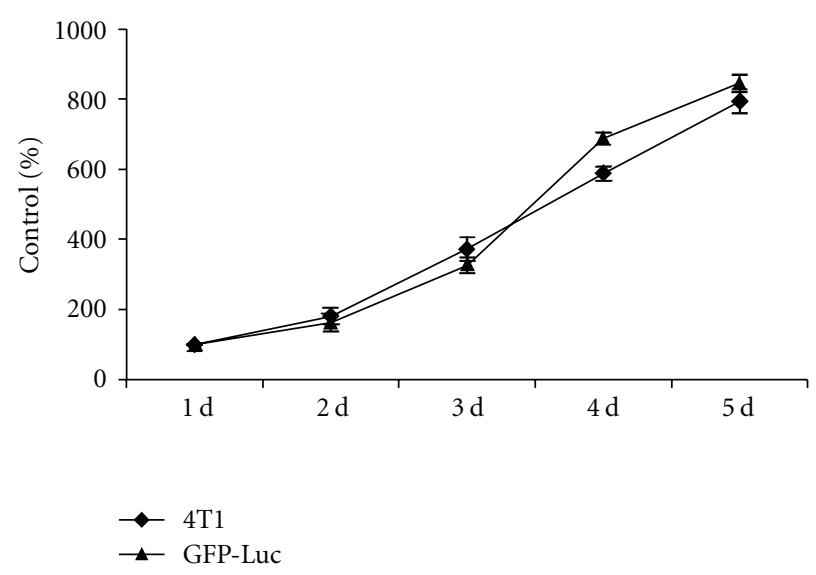

(a)

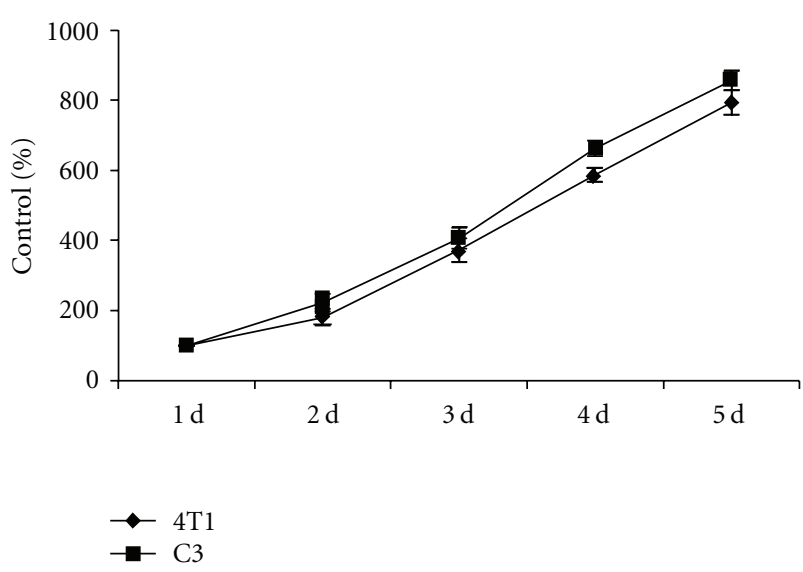

(b)

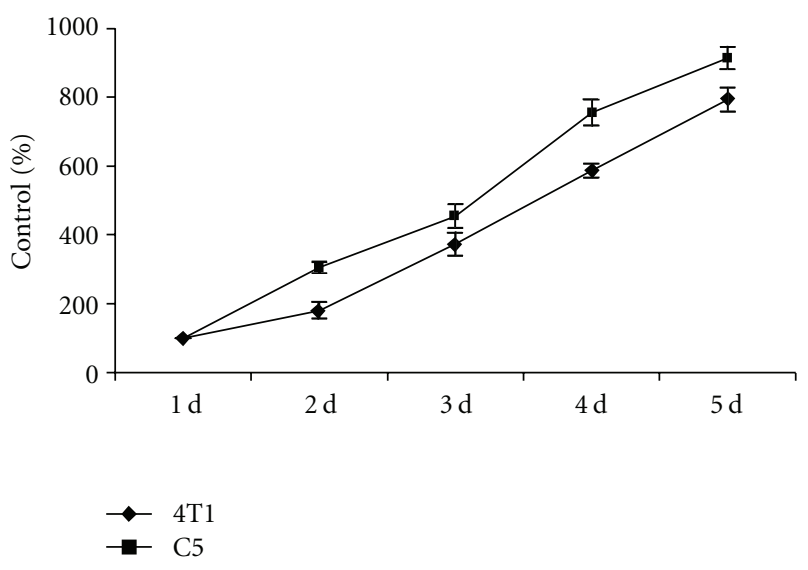

(c)

FIGURE 3: Comparison of cell proliferation between the parental 4T1 and three of its derivatives. 4T1 cells and its derivatives ((a) GFP-Luc; (b) C3; (c) C5) were seeded in 96-well plates and the proliferation was quantitated using the MTS assay on a daily basis for a period of 5 days. The values are means \pm S.D. of three independent experiments.

into a piggyBac transposon construct. PiggyBac has shown efficient integration of genes into cellular chromosomes at multiple foci when it is cointroduced with transposase [10]. The new construct was designated pIR-HER2-puromycin (Figure 1(a)). In addition, another transposon construct, pIR-GFP-Luc, that contains a fusion gene comprised of green florescent protein (GFP) and luciferase genes (Figure 1(b)), was also constructed. This construct was used in combination with other constructs as a convenient marker for identification of the stably transfected cells. $4 \mathrm{~T} 1$ cells were cotransfected with either a triple combination of pIR-HER2puromycin, pIR-GFP-Luc, and pCMV-piggyBac (containing the gene encoding the transposase) or a double combination of pIR-GFP-Luc and pCMVpiggyBAC (for making a control 4T1 containing the GPF marker gene only). The transfected cells were selected for both puromycin and GFP expression and then cloned as described in Section 2.

To confirm that they express HER2, the selected clones were subjected to Western blot analysis using an antibody specific for HER2. As shown in Figure 2, all the five cloned cell lines (C1-C5) selected from the triple transfection show a high level of HER2 expression, while the single-cell line derived from the double transfection without pIR-HER2puromycin (designated GFP-Luc) was negative for HER2. This result was also confirmed by FACS analysis after the cells were stained with FITC-conjugated antibody specific for HER2 (data not shown). These results suggest that cointegration efficiency is extremely high with the TTAA-specific transposon piggyBac system when multiple components are codelivered. Part of the reason for such a high efficiency in multiple gene co-integration is probably due to high copy number of gene integration during piggyBac-mediated gene transduction. It has been estimated that, by average, there are 50 copies of gene integration at multiple chromosome foci when using the piggyBac gene delivery $[14,15]$.

The ability of the piggyBac system to integrate multiple genes at once in a versatile way has a clear advantage over other commonly used gene integration vectors such as adenovirus-associated viral vector, retroviral, and lentiviral vectors. Additionally, the high frequency of gene integration and lack of overproduction inhibition may have also contributed to the relatively high level of gene expression from cells established from this method than with other methods [10]. Indeed, all the cell lines that we have established, from 


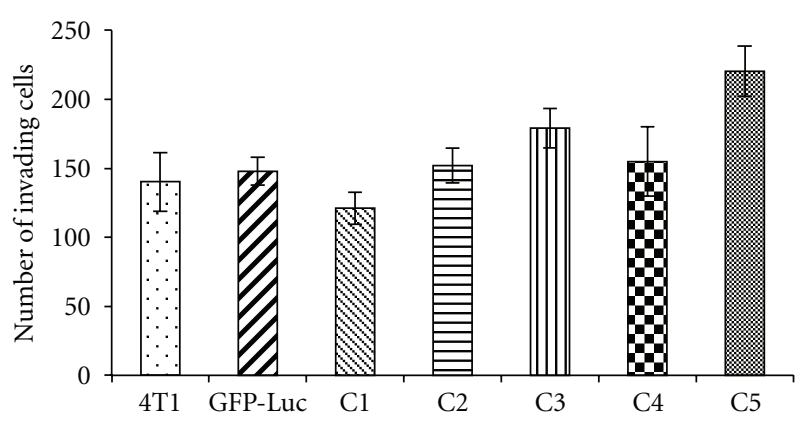

(a)

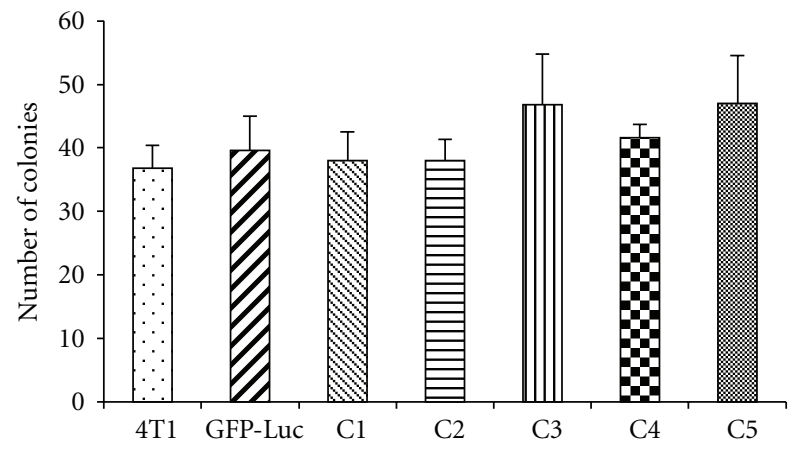

(b)

Figure 4: Comparison of invasion and colony formation between the parental 4T1 and its derivatives. (a) Matrigel invasion assay. Cells were seeded in BioCoat Matrigel invasion chambers and cell invasion was evaluated $24 \mathrm{~h}$ later by counting five adjacent microscope fields at 20x magnification for metastatic cells clinging at the bottom of the membrane. Columns are means \pm S.D. of three independent experiments. (b) Indirect independent growth measurement of cells for single-cell colony formation. Cells were seeded in 6-well plates in soft agar and cell colonies were determined 20 days later. Columns are means \pm S.D. of three independent experiments.

C1-C5, show very strong GFP expression. This method is now routinely used in our lab for making stable cell lines.

3.2. In Vitro Characterization of the Established Cell Lines. We initially compared the newly established 4T1 cell lines with the parental line for their growth characteristics. We chose two cell lines, C3 and C5, out of the five established colonies. The same number of cells were seeded in different wells and harvested at different times. The cell number was counted and compared. The results show that GFP-Luc and C3 grew at the same rate as the parental 4T1 cells (Figures 3(a) and 3(b)), while C5 grew at a slightly faster rate (Figure 3(c)): there were $87 \%$ more C5 cells than $4 \mathrm{~T} 1$ on day $2,37 \%$ more on the day $3,31 \%$ more on the day 4 , and $20 \%$ more on the day 5. Overall these results show that expression of the HER2 gene in 4T1 cells did not dramatically change the growth properties of the cells.

Since metastasis is a key feature of 4T1 tumor cells when implanted in animals, we next compared the newly established cell lines with the parental line for their in vitro matrigel invasive capability. Cells were set up in 24-well

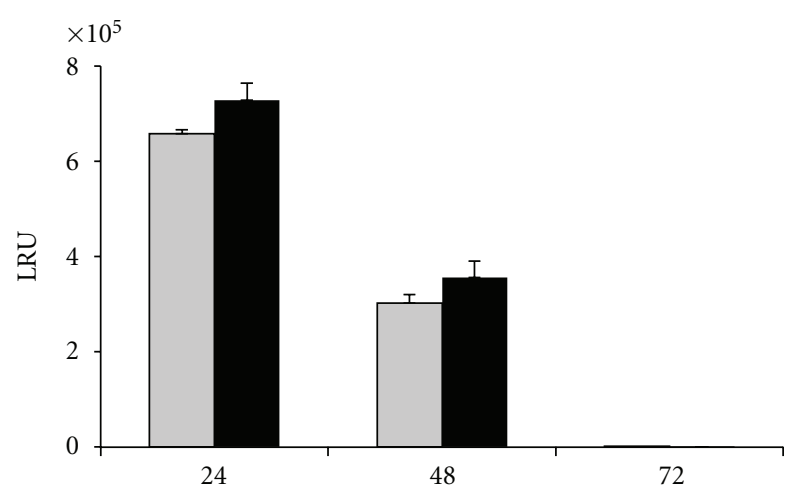

(h)
$\square \mathrm{C} 3$
- $\mathrm{C} 5$

(a)
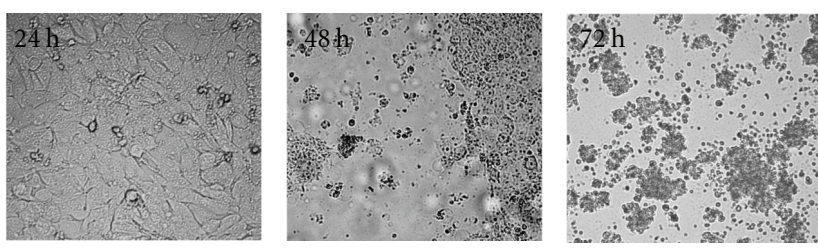

(b)

Figure 5: Cytolytic activity of T-effector cells against HER2expressing 4T1 derivatives. C3 and C5 cell lines were chosen for this experiment. (a) Cytolytic activity as measured by the luciferase release (as LRU for luciferase release unit) after the HER2expressing C3 and C5 cells was mixed with the T-effector cells that had been transduced with a chimeric T-cell receptor that can specifically recognize HER2. Values are means \pm S.D. of three independent experiments. (b) Morphology of C3 cells during the course of incubation with the T-effector cells.

BioCoat Matrigel invasion chambers and incubated for $24 \mathrm{~h}$ for the invasive measurement by counting the number of invading cells. Results in Figure 3(a) show that most of the HER2-expressing cells $(\mathrm{C} 1, \mathrm{C} 2$, and $\mathrm{C} 4)$ maintain the same invasive capability as the parental 4T1 cells, while the invasiveness of C-3 and C-5 is slightly enhanced.

We also compared the independent growth of the newly established cells with the parental 4T1 line. Our results show that colonies 3 and 5 grew approximately 30\% faster than the parental cell line and the GFP-Luc control cell line (Figure 4(b)). For the rest of the cells, no significant difference for the growth rate was detected when compared with the parental $4 \mathrm{~T} 1$ cells. These results are consistent with reports from other groups that demonstrate that introduction of human HER2 gene into murine mammary tumor cells does not dramatically alter cell growth and metastatic properties [16].

3.3. The Newly Established Cell Lines Can Be Efficient Recognized by HER2-Specific T Lymphocytes. To determine if the introduced HER2 in the newly created 4T1 cells can serve as an effective tumor antigen, we used these cells as targets to measure the cytolytic activity of $\mathrm{T}$ lymphocytes that can specifically recognize HER2-expressing tumor cells. 


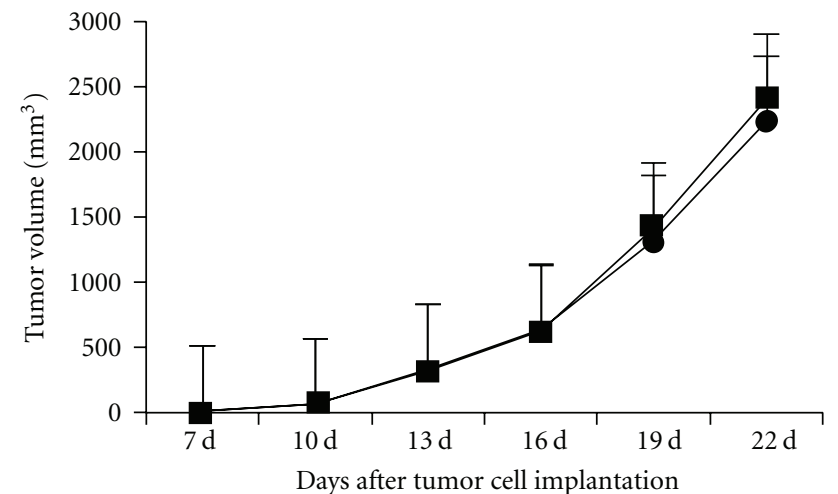

$-4 \mathrm{~T} 1$
- GFP-Luc

(a)

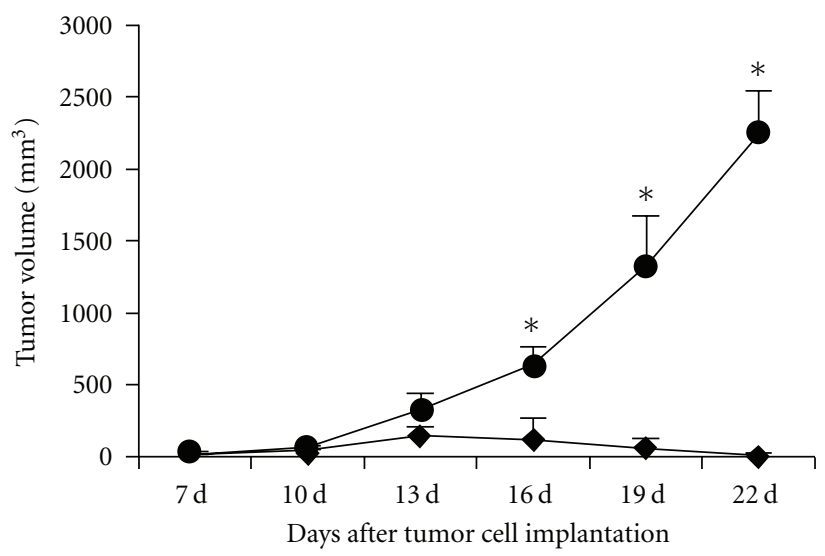

$-4 \mathrm{~T} 1$

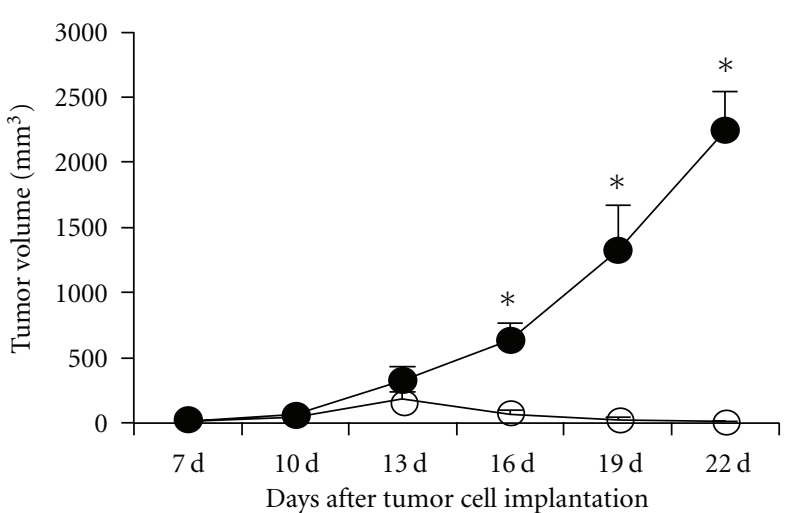

$-4 \mathrm{~T} 1$ (b)

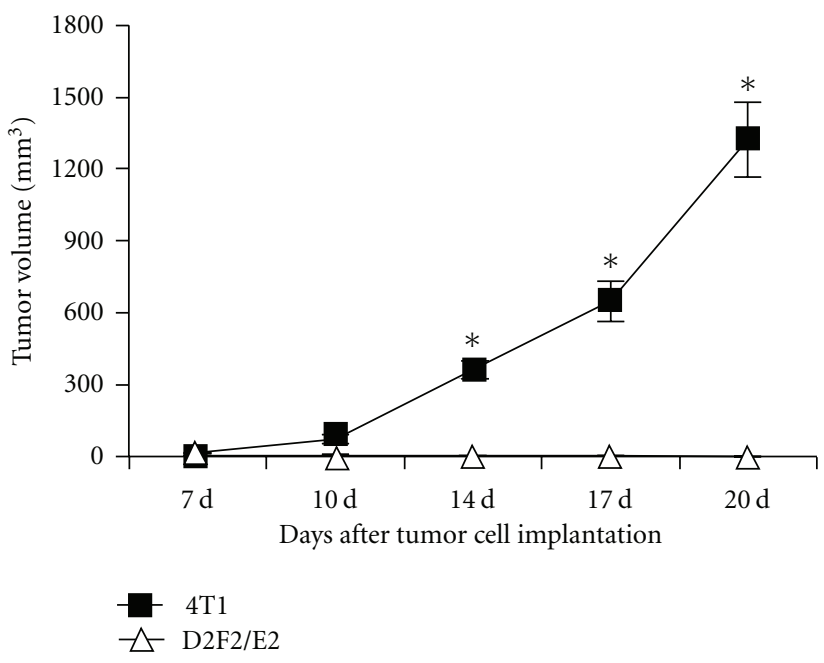

(d)

(c)

FIGURE 6: Tumor formation and growth from the initially implanted and subsequently rechallenged tumor cells. (a) Comparison of tumor growth between $4 \mathrm{~T} 1$ parental cell line and the established GFP-Luc that does not express HER2. (b) and (c) Comparison of tumor growth between 4T1 parental cell line and the established HER2-expressing C3 (b) and C5 (c) lines. (d) Tumor growth from the rechallenged 4T1 or D2F2/E2 cells in mice that had the original C3 or C5 tumor disappeared. ${ }^{*} P<0.01$ versus $4 \mathrm{~T} 1$ tumor.

These modified $\mathrm{T}$ lymphocytes contain a chimeric antigen receptor comprising a single chain antibody specific to HER2 [11]. The cytolytic activity was measured with a recently developed nonradioactive method in our lab [13], which fully exploits the high sensitivity and the relative simplicity of luciferase quantitative assay. The results show that incubation of both clone 3 and 5 with the T-effector cells caused the progressive loss of the intracellular marker GFP-Luc. After a $72 \mathrm{~h}$ of incubation, luciferase activity was reduced to an almost undetectable level (Figure 5(a)). The cell morphology also showed the progressive development of cytopathic appearance of the cells, and nearly all the cells showed abnormal morphology by $72 \mathrm{~h}$ after incubation, consistent with the quantitative luciferase measurement in Figure 5(a). These results suggest that the introduced HER2 can indeed serve as the desirable antigen for recognition by the modified T-effector cells.
3.4. Tumor Formation of the Established Cells in Immune Competent Mice. Next, we orthotopically implanted some of the established lines into immune competent syngeneic Balb/c mice (into the second mammary fat pad). The parental 4T1 and GFP-Luc cells were included in this experiment as controls. For the first 2 weeks, all the implanted tumors cells formed tumors almost equally efficiently. However, after the second week, tumors formed from implantation of C3 and C5 started to shrink and gradually disappeared by the third week (Figures 6(b) and 6(c)), while tumors derived from the parental 4T1 cells and the control GFP-Luc cells grew continuously (Figure 6(a)).

Since tumor growth from GFP-Luc and 4T1 lines was similar and only the tumors derived from HER2-expressing C3 and C5 cells were gradually disappearing, one explanation is that the tumor bearing mice in the C 3 and C5 implantation groups had developed an immune response that was specific 
to HER2. To examine this possibility, we randomly divided the tumor-free mice from the C3 and C5 implantation groups and challenged them with either the parental $4 \mathrm{~T} 1$ cells or D2F2/E2 cells, an established murine mammary tumor line that also constitutively expresses HER2 and is syngeneic to Balb/c mice [16]. While implantation of $4 \mathrm{~T} 1$ cells developed into sizeable tumors, D2F2/E2 cells failed to initiate palpable tumors during the entire period of this experiment (up to 3 weeks after tumor implantation).

Together these results show that, despite the highsequence homology between the human HER2 and murine neu genes, the former can be presented as a tumor antigen when introduced into the murine 4T1 cells which subsequently induces an immune response that results in tumor disappearance of the initially formed tumors. This result is different from other murine tumor cells that have been transduced with the human HER2 gene, which seem to be able to form tumors and the tumor growth can be sustained for a reasonably long period of time $[16,17]$. Part of the reason for such a discrepancy is probably because 4T1 cell line is more aggressive than other tumor cells. Rapid growth and efficient metastasis of $4 \mathrm{~T} 1$, especially its ability to naturally metastasize through the lymphoid system [2], may have contributed to the apparently stronger immunity of HER2 in this model than in other syngeneic murine tumor models.

3.5. Tumor Formation of the Newly Established Cells in Immune Compromised Mice. Next, we orthotopically implanted C3, C5, and the parental 4T1 cells into different groups of immunodeficient mice. Both C3 and C5 cells formed tumors at the same time as the 4T1 cells. Moreover, unlikely what have been detected in the immune competent animals (Figures 6(b) and 6(c)), the tumors derived from C3 and C5 cells showed the same growth curve as that from the parental 4T1 cells until the end of this experiment when tumors from all the groups reached a relatively large size (Figure 7). Examination of the organ tissues from these mice after the termination of the experiment revealed that distant metastatic tumor nodules were frequently detectable in lymph nodes, lung, and liver, and the quantity and pattern are similar to those seen from the $4 \mathrm{~T} 1$ group (data not shown).

Recent studies have shown that engraftment of naive $\mathrm{T}$ lymphocytes to express tumor antigen-specific chimeric immune receptors is an attractive avenue for providing large numbers of effector cells. These T-effector cells recognize the tumor cells in a MHC nonrestriction way and adoptive transfer of these T-killer cells into tumor-bearing hosts represents an attractive immunotherapy approach [18]. Our in vitro studies show that the newly established C1-C5 cells can serve as excellent targets for T-effector cells that have been transduced with a chimeric T-cell receptor and specifically recognize HER2-expresing tumor cells through a single-chain antibody. The efficient tumor formation of these cells in immunodeficient mice and the close resemblance of the metastatic pattern of the parental line suggests that they can provide an adequate tumor model for studies such as the

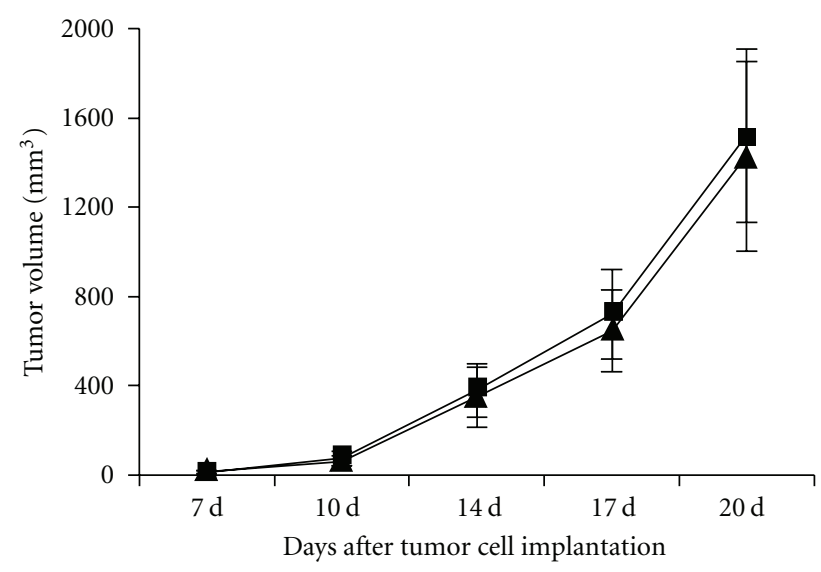

(a)

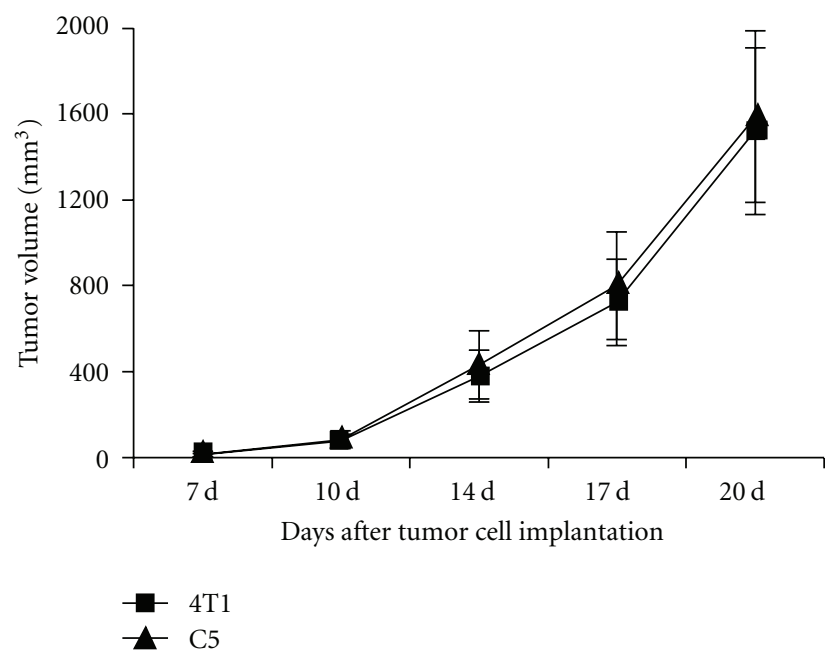

(b)

FIgure 7: Tumor growth of C3 and C5 cells in immunocompromised mice. (a) Comparison of tumor growth between 4T1 parental cell line and the established HER2-expressing C3 cells. (b) Comparison of tumor growth between 4T1 parental cell line and the established HER2-expressing C5 cells.

chimeric adoptive transfer of humoral or cellular immunities specific for HER2.

\section{Conclusions}

In this study, we stably transduced the HER2 tumor antigen into a popular murine breast cancer model. In vitro studies show that $\mathrm{T}$ cells transduced with a chimeric antigen receptor comprising a single-chain antibody specific to HER2 can efficiently recognize and kill the newly established $4 \mathrm{~T} 1$ cells. When implanted into immune competent mice, these tumor cells stimulated an effective anti-HER2 immune response that can effectively shrink and then eradicate the initially established tumors. We thus conclude that these new 4T1 cell lines are useful for both in vitro and in vivo studies in 
characterizing immunotherapeutic approaches in a tumor model that closely resembles human disease.

\section{Conflict of Interests}

The authors declare no conflict of interests.

\section{Acknowledgments}

The authors thank Dr. Matthew Wilson for providing the piggyBac construct and Drs. Fred Miller and Wei Zhen Wei for providing $4 \mathrm{~T} 1$ and D2F2/E2 cell lines. This work was supported in part by NIH/National Cancer Institute grants R01CA106671 and R01CA132792 (to XZ).

\section{References}

[1] I. Crnic and G. Christofori, "Novel technologies and recent advances in metastasis research," International Journal of Developmental Biology, vol. 48, no. 5-6, pp. 573-581, 2004.

[2] C. J. Aslakson and F. R. Miller, "Selective events in the metastatic process defined by analysis of the sequential dissemination of subpopulations of a mouse mammary tumor," Cancer Research, vol. 52, no. 6, pp. 1399-1405, 1992.

[3] K. Tao, M. Fang, J. Alroy, and G. G. Gary, "Imagable 4T1 model for the study of late stage breast cancer," BMC Cancer, vol. 8, article 228, 2008.

[4] M. Nakamori, X. Fu, R. Rousseau, S. Y. Chen, and X. Zhang, "Destruction of nonimmunogenic mammary tumor cells by a fusogenic oncolytic herpes simplex virus induces potent antitumor immunity," Molecular Therapy, vol. 9, no. 5, pp. 658-665, 2004.

[5] H. Li, A. Dutuor, X. Fu, and X. Zhang, "Induction of strong antitumor immunity by an HSV-2-based oncolytic virus in a murine mammary tumor model," Journal of Gene Medicine, vol. 9, no. 3, pp. 161-169, 2007.

[6] D. Schadendorf, I. Fichtner, A. Makki et al., "Metastatic potential of human melanoma cells in nude mice-characterisation of phenotype, cytokine secretion and tumour-associated antigens," British Journal of Cancer, vol. 74, no. 2, pp. 194-199, 1996.

[7] R. E. Roses, E. C. Paulson, A. Sharma et al., "HER-2/neu overexpression as a predictor for the transition from in situ to invasive breast cancer," Cancer Epidemiology Biomarkers and Prevention, vol. 18, no. 5, pp. 1385-1389, 2009.

[8] E. Tagliabue, A. Balsari, M. Campiglio, and S. M. Pupa, "HER2 as a target for breast cancer therapy," Expert Opinion on Biological Therapy, vol. 10, no. 5, pp. 711-724, 2010.

[9] M. P. Piechocki, Y. S. Ho, S. Pilon, and W. Z. Wei, "Human ErbB-2 (Her-2) transgenic mice: a model system for testing Her-2 based vaccines," Journal of Immunology, vol. 171, no. 11, pp. 5787-5794, 2003.

[10] M. H. Wilson, C. J. Coates, and A. L. George Jr., "PiggyBac transposon-mediated gene transfer in human cells," Molecular Therapy, vol. 15, no. 1, pp. 139-145, 2007.

[11] N. Ahmed, M. Ratnayake, B. Savoldo et al., "Regression of experimental medulloblastoma following transfer of HER2specific T cells," Cancer Research, vol. 67, no. 12, pp. 59575964, 2007.

[12] A. C. Borley, S. Hiscox, J. Gee et al., "Anti-oestrogens but not oestrogen deprivation promote cellular invasion in intercellular adhesion-deficient breast cancer cells," Breast Cancer Research, vol. 10, no. 6, article R103, 2008.
[13] X. Fu, L. Tao, A. Rivera et al., "A simple and sensitive method for measuring tumor-specific T cell cytotoxicity," PLoS ONE, vol. 5, no. 7, Article ID e11867, 2010.

[14] D. L. Galvan, Y. Nakazawa, A. Kaja et al., "Genome-wide mapping of piggybac transposon integrations in primary human T cells," Journal of Immunotherapy, vol. 32, no. 8, pp. 837-844, 2009.

[15] Y. Nakazawa, L. E. Huye, G. Dotti et al., "Optimization of the piggybac transposon system for the sustained genetic modification of human T lymphocytes," Journal of Immunotherapy, vol. 32, no. 8, pp. 826-836, 2009.

[16] M. P. Piechocki, S. A. Pilon, C. Kelly, and W. Z. Wei, "Degradation signals in ErbB-2 dictate proteasomal processing and immunogenicity and resist protection by cis glycine-alanine repeat," Cellular Immunology, vol. 212, no. 2, pp. 138-149, 2001.

[17] M. L. Penichet, P. M. Challita, S. U. Shin, S. L. Sampogna, J. D. Rosenblatt, and S. L. Morrison, "In vivo properties of three human HER2/neu-expressing murine cell lines in immunocompetent mice," Laboratory Animal Science, vol. 49, no. 2, pp. 179-188, 1999.

[18] R. A. Morgan, M. E. Dudley, and S. A. Rosenberg, "Adoptive cell therapy: genetic modification to redirect effector cell specificity," Cancer Journal, vol. 16, no. 4, pp. 336-341, 2010. 


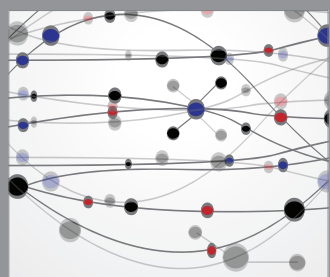

The Scientific World Journal
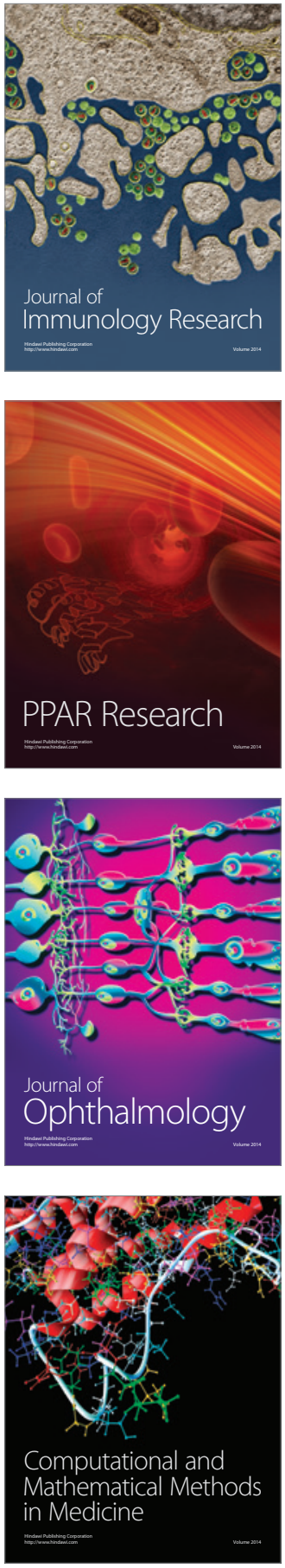

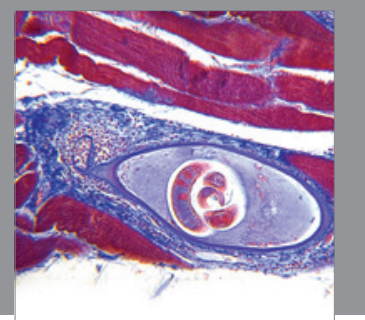

Gastroenterology

Research and Practice
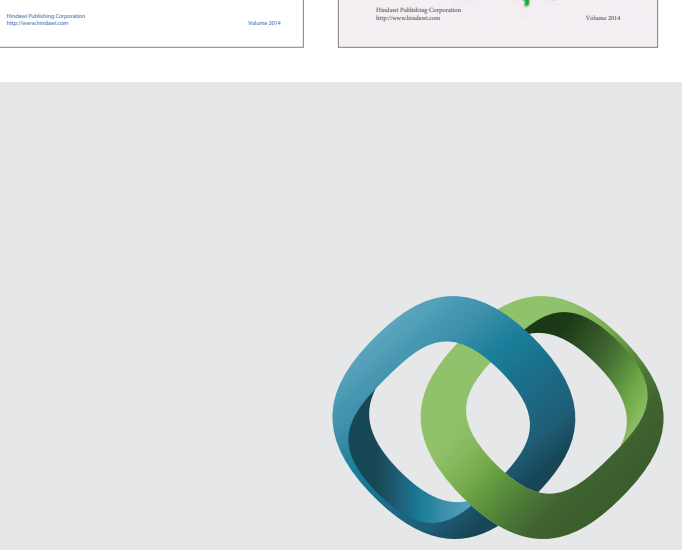

\section{Hindawi}

Submit your manuscripts at

http://www.hindawi.com
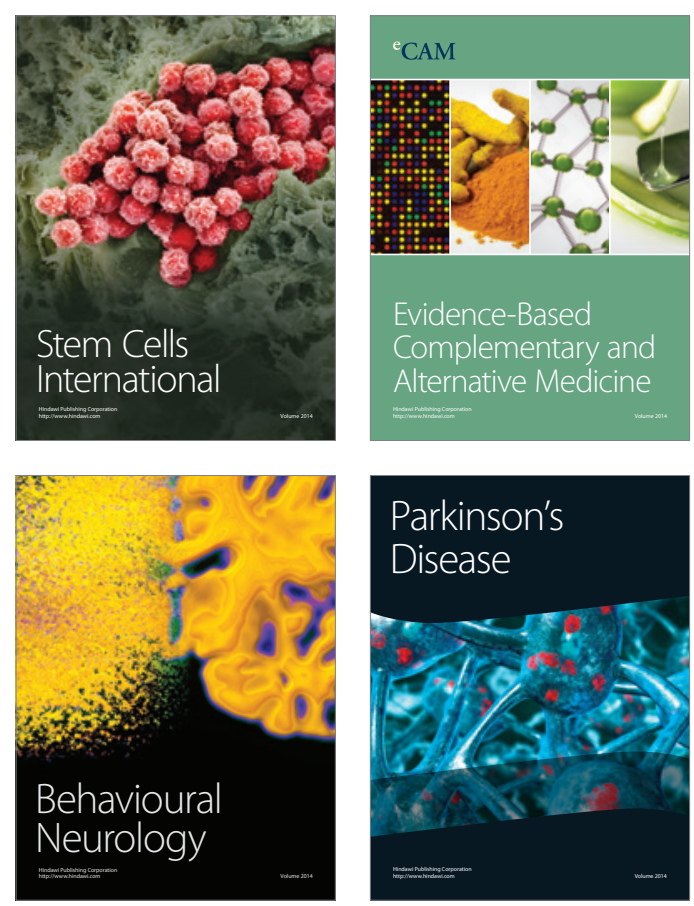

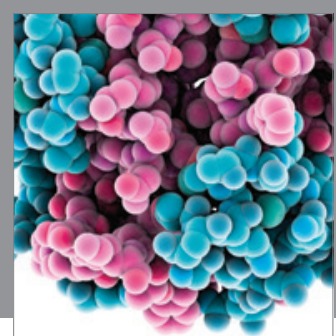

Journal of
Diabetes Research

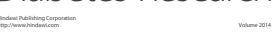

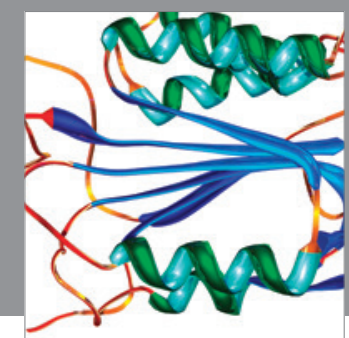

Disease Markers
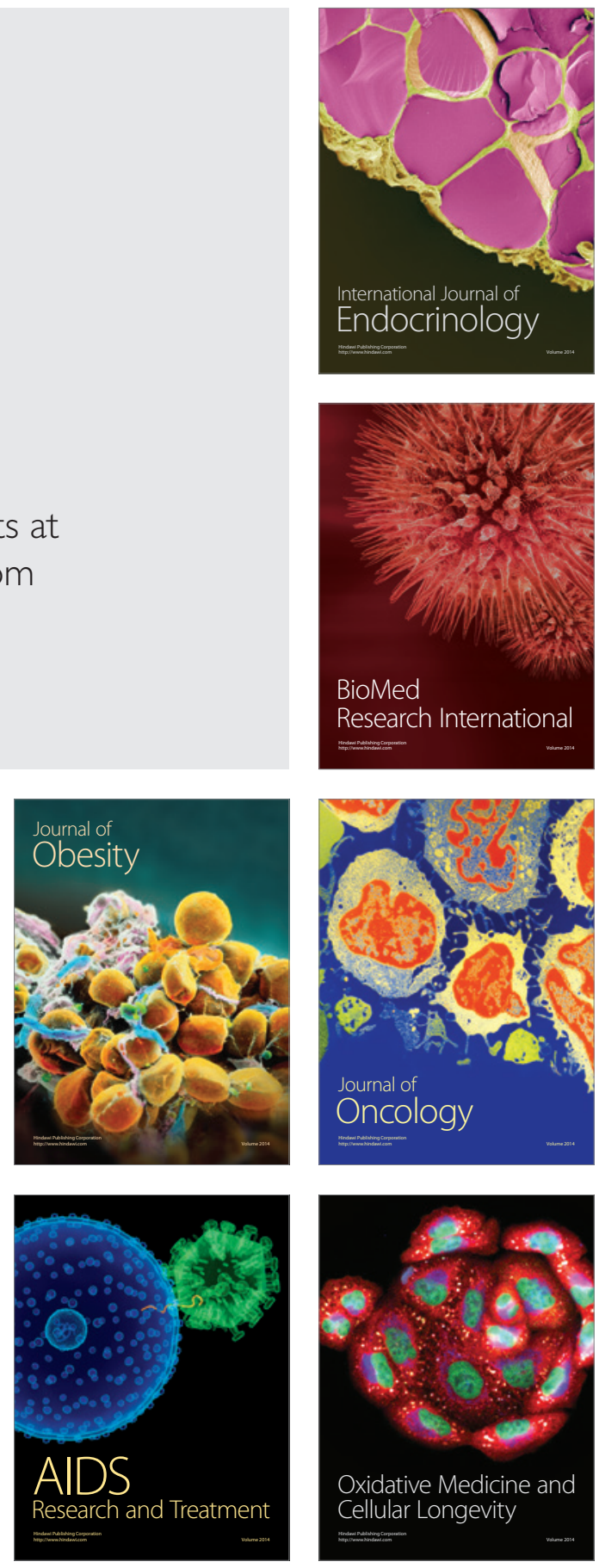